\title{
Water oxidation with holes: what we learn from operando "synchrotron" studies (Conference Presentation) (Withdrawal Notice)
}

\section{Artur Braun}

Artur Braun, "Water oxidation with holes: what we learn from operando " synchrotron" studies (Conference Presentation) (Withdrawal Notice)," Proc. SPIE 9935, Solar Hydrogen and Nanotechnology XI, 99350D (3 November 2016); doi: 10.1117/12.2238802

Event: SPIE Optics + Photonics for Sustainable Energy, 2016, San Diego, California, United States 


\section{Water oxidation with holes: what we learn from operando "synchrotron" studies (Conference Presentation) (withdrawal notice)}

Proc. SPIE 9935, 99350D (2016)

Online Publication Date: 3 November 2016

Withdrawn from Publication: 8 November 2016

Conference Date: 29-31 August 2016

Conference Location: San Diego, California, United States

Conference Title: Solar Hydrogen and Nanotechnology XI

Conference Chairs: Chung-Li Dong

Artur Braun

Empa (Switzerland)

This conference presentation, originally published on 3 November

2016, was withdrawn per author request. 